Article

\title{
Applications of Non-Standard Analysis in Topoi to Mathematical Neuroscience and Artificial Intelligence: Infons, Energons, Receptons (I)
}

\author{
Ileana Ruxandra Badea ${ }^{1}$, Carmen-Elena Mocanu ${ }^{2}$, and Ovidiu Păsărescu ${ }^{3 *}$ \\ ${ }^{1}$ The Bucharest University of Economical Studies, The Faculty of Economic Cybernetics, Statistics \\ and Informatics
}

15-17 Dorobanti Avenue, District 1, Bucharest, Romania; badea_ruxandra@yahoo.com,

${ }^{2}$ Carol Davila University of Medicine and Pharmacy, Faculty of General Medicine

8, Eroii Sanitari Avenue, District 5, Bucharest, Romania; Carmen.Mocanu@ stud.umfcd.ro,

${ }^{3}$ Simion Stoilow Institute of Mathematics of the Romanian Academy

21, Calea Grivitei Street, 010702 Bucharest, Romania; Ovidiu.Pasarescu@imar.ro

* Author to whom correspondence should be addressed; E-Mail: Ovidiu.Pasarescu@imar.ro; Tel: (+40) (0) 2131965 06; Fax: (+40) (0) 213196505

\begin{abstract}
This work promotes new methods in Mathematical Modeling, consisting in the use of the methods of Non-standard Analysis in Topoi, having as its main purpose, the mathematical definitions of the pseudoparticles from the title, with arguments from Biology/Physiology (Mathematical Neuroscience), Physics (String Theory and Emergent Quantum Mechanics), and Cybernetics (Global Brain, including Natural and Artificial Intelligence). The connections between brain and mind will be scketched via the genetic/epigenetic interplay. The topoi model the intuitionistic logic (multi-valued) and have been used in Quantum Physics while Non-Standard Analysis in SET (= the Category of sets) has been applied in Mathematical Economics; topics from Theory of Categories were also used in the study of Consciousness; however, the combination topoi - non-standard analysis was never used until now in Applied Mathematics. Another important objective is to produce progress in this aria of Pure Mathematics also - the build of non-standard analysis in classes of topoi, already used in Physics. We propose the logic of non-standard extensions in topoi as a model of the human thinking (based on infons/receptons), these theories representing new top and very difficult results in Abstract Mathematics either.
\end{abstract}


Keywords: non-standard analysis, topos theory, artificial intelligence, brain studies, intuitionistic logic

Classification: MSC 18D35, 16B50, 16B70, 18F10, 03B50, 26E35

\section{Introduction}

Preliminary note This is the version 2 of the paper [25] from the References, whose Section 9., entirey written by Dr. Florin Nichita, became part of Section 3. from https://arxiv.org/pdf/2002.05345.pdf, and has been submitted for publication. Noreover, the Section 4. of the present version is new. This generated a change of authors. The remaining part of this version is a shortened form of the previous one, to which we make many references.

This present paper contains both known and new results, because it addresses to an auditorium of a great diversity: neuroscientists, informaticians, physicians, physicists, biologists, mathematicians,... so we tried to write a paper which could be read by different kind of specialists. Even the authors and their collaborators belong to various scientific areas (neuroscience, mathematics, medicine, economics; professors, researchers, students). We hope that we produced an enough self-contained and readable paper by many categories interrested in the topics. In the same time, the preprint [25] represents a much more expanded version of this article, from mathematical point of view, collecting most of what we considered as being the necessary mathematical notions. So, the paper shortly re-explain already known notions and results, and it also contains new and original contributions. We present them in what follows. The main original contribution is the idea of considering the non-standard extension of the intuitionistic logic (in topoi) as modeling the human thinking, consciousness, and considering together both natural and artificial intelligence, as parts of the global brain, as explained in Sections 2., 3., and 4. We also contribute to the development of the Non-stardad Analysis in set-type exponential topoi, in the aria of Pure Mathematics, see Theorem 6.2.5. Information and energy represent the two facets of an elementary particle in String Theory (the string vibrates = energy in a specific way (amplitude, frequence, spin,...) = information, in order to give one or another one of the elementary particles). They do not reduce to our brain, neither to our body, they travel freely realising connections between the interior (brain, senzory organs, DNA = genetics) and the exterior (epigenetics) of the human body, generating the mind, reciprocically related to the brain (see [38], [10]). We deal with these subjects in more detail in Sections 2., 3., and 4. In Sections 5. and 6. we recall the basic notions of our modeling approach (based on Topos Theory and its Non-standard extension), the examples from 5.2.6. - 5.2.8. being original. The interpretation of theories (from mathematical logic, model theory) and of the corresponding categories (models of the theories) as infons, energons, and receptons represents an original approach too. For details concerning these things, see Sections 5. and 7. However, this paper shortly uses some notions, not explicitely introduced here, as: (Quantum)Neural Networks, (Quantum)Turing machines, and further 
developments of the Non-standard Analysis in more general topoi. All these things will be considered in a forthcoming paper.

For Topoi Theory we refer to the book [13]. For Model Theory we refer to [7]. For non-standard analysis in the topos SET we refer to the book [14].The topoi model the intuitionistic logic (multi-valued) and have been used in Quantum Physics (see [12] and its references; see also [8]) while Non-Standard Analysis in SET (introduced by A. Robinson [35]) has been applied in Mathematical Economics (see [1] and its references). We propose the logic of non-standard extensions in topoi as a model of the human thinking (based on infons) inspired by the paper [18]. These theories represent top and very difficult results in Abstract Mathematics. We also refer to [10], [17], [36], [38], [41]. Other References will appear later.

All the subjects of this paper are considered in order to justify, both from the point of view of Neuroscience, Cybernetics, and from the view od Physics, the introduction of the pseudoparticles called by us Infons and Receptons, with Energons between them, as a Corpus Callosum in the Brain: to justify how they should be (Sections 2.-4.), and to built mathematically such things (sections 5.-7.).

\section{Informatio/Reception : Brain/Mind, Genetics/Epigenetics}

In this Section we start to put in evidence, using known and, by many experts recognized results from Biology/Physiology, which relates between them the Information and its Reception with Energy (see [39], [10], [38]).

2.1. Information and Entropy. First of all, information is related to organisation. We say that some systems or objects are more complex than others because we perceive them as containing more information. For example, it is obvious that an epithelial tissue contains more information than a cell. Epithelial cells exhibit distinct polarity, having an apical, a lateral and a basal domain. This organisation involves more information, as the molecular mechanism responsible for this arrangement is required to create a functional barrier between adjacent cells. Following Tom Stonier's idea from [39], information is related to entropy, because the latter is a measurement of organisation. They are exponentially inverse proportional: as a consequence, if - inside a complex system - the entropy decreases, meaning that matter is more organised, the amount of incorporated information increases. We think it is really important to see how this new concept - the incorporated information - influences our lives and more specifically the role it plays in the structure of matter. How do atoms know how to bind together and form a more complex structure? How can a simple stem cell differentiate in so many other types of cells? Information dictates all those processes. Everything contains infons (see section 7, for their mathematical definition, one of the main purposes of this paper). Let's take now, as an example, a sound wave. We can tell for sure that it contains some kind of information, that travels through air until it reaches the human ear. Here it is transformed into a nervous impulse and travels to the brain, where it will be perceived. A sound wave is a mechanical wave that causes local regions of compression and rarefaction through the medium, deviating the local pressure from the equilibrium pressure. Therefore, sound, which is made of information, changes the structure of matter. In neuroscience it is of extreme interest to see how information affects the brain. We, as humans, are born with unconnected neurons. In the first two years, we form synapses while experiencing the world around us. As we grow up, we lose almost $50 \%$ of the 
already formed synapses, but we also form others while interracting with the environment. How does information influence this process? The more we interact with something, the more powerful the synapse gets. It's like a road through a forest. At first, there's nothing there, but as more and more people go along it, the grass disappears and a path is formed. The same thing happens in the brain. We believe that information extends one's life. During the first part of our lives, the human organism functions in an anti-entropic way. As it gets more organised, the entropy decreases, instead of increasing. However, after this period, the entropy of the human organism continues to increase until it reaches a state of equilibrium with the environment, when the organism dies, because no more energy exchange can be done. By increasing the amount of information one takes in, the entropy of the organism decreases, meaning that it prolongs the process of ageing. Thus, learnitheng new things delays the decay of the brain primarily, but of other organs as a consequence as well. For a much more physico-mathematical approach of such kind of things, see Sections 3. and 7. of this paper.

2.2. Information and Reception, Brain and Mind. For further details related to this paragraph, we refer to the books [10], [38]. The Brain is the main organ where the mind is born, but the brain is far from being the only entity involved in the growth of the human mind. The mind is a by-product of the neural networks from the brain (synapses), in continous transformations (some synapses disappear while new ones are born), representing the so-called plasticity of the brain. The mind is a by-product of other kind of communications between neurons (and the glial environment), namely the quantum networks connected by the so-called microtubules. Certainly, this interactions are between the brain, through the sensory organs, moderated by the information from the DNA molecule (genetics), and the environment (epigenetics). The human being has much information in its DNA, but only some of it will be used in a life-long period. Simplifying, there is information that tells us to start trembling when it is cold (or to sweat when it is hot). However, if we spend a life-time period at the Ecuator we will never experience the trembling (respectively, if we spend a life-time period at the North pole we will never experience sweating). So, the information from the around $2 \mathrm{~m}$ long DNA molecule is read by the environment epigenetics, the system genetics/epigenetics becoming a kind of (quantum) Turing machine, generating, in particular, the evolution of the mind, which, through plasticity, generates the evolution of the brain, and vice-versa.

We - the humans - think using both consciousness (awarness) and subconsciousness. The Logic of Consciousness is intuitionistic (we have, mainly, feelings about things, like about the truth of Conjectures in Mathematics, as an example), and the Reunion between Consciousness and Subconsciousness is a conservative extension of consciousness (that is, the subconsciousness does not alter our awarness). So, the logic of human thinking is very similar to the logic of infons from Section 7., having as models non-standard objects from non-standard enlargements of topoi - see Section 7. again (based on Sections 5. and 6. also). From a mathematical point of view, it is necessary to understand how the (quantum) neural networks from the brain (synapses) are influenced by the genetic-epigenetic (quantum) Turing mashine. The Quantum Physics approach is better to be based on truth values than on probabilities, the truth values-approach being used in topoi (see [8]). So, the reunion between consciousness and subconsciousness is modeled by the non-standard logic in enlarged topoi (the IIST logic from section 7. as the Theory of Infons, with models - the Cathegories of Receptons - as explained in the forthcoming Section 7.). 
And, another important thing. A Theorem of Mathematics (for example) is based on the work of many others, so it is a by-product of the global brain. The Global Brain includes all the human brains AND technology, used both for getting information from the outside of the direct human perception, and for connecting the brains (libraries, social networks, ...) - see section 4. In this case the genetic-epigenetic interaction is between the Global Brain and the Environment, generating a kind of Global Plasticity for the Global Brain, generating a Global Mind (for some details, see Section 4.).

2.3. = 3.2. Information, Energy, and Reception. This paragraph could be introduced here, but it can also belog to the next Section 3., and we prefered to put it there (it is mainly related to Physics, while this Section is mainly related to Biology/Physiology).

\section{Information/Reception : String Theory/Emergent Quantum Mechanics}

In this Section continue to put in evidence the relation Information - Energy - Reception, using known and, by many experts recognized results from Celestial Mechanics/Physics (see [41], [17], [44], [5]).

3.1. Max Tegmark's Multiverses. Certainly, everything happens in our real world, our real Universe, or our real Multiverse, or... This means that the human brain, human being, global brain,... represent substructures of the highest structure which must exist. There are discussions on what this highest structure is. We recall here the Theory of Multiverses belonging to Max Tegmark (see [41]). Namely, he classifies the notions of Multiverse in 4 categories. First of all, what is an Universe. In these theories the Universe is, by definition, the visible Universe. It is a 3D - sphere having its radius of about 14 billion light-years (the approximate period that has passed from the Big-Bang). This sphere is increasing as time passes. A rank 1 Multiverse is a family of Universes which could merge in some future in a bigger Universe. However, the General Relativity does not forbid that the space dilate with a speed higher than the speed of light. This means that two rank 1 Multiverses separated by a volume dilating faster than the speed of light will never merge at the level of a visible Universe. Such a family of rank 1 Multiverses is a rank 2 Multiverse. Because the evolutions are independent to a certain extent, the Universal constants, and even some of the laws of Physics among the rank 1 Multiverses belonging to the rank 2 Multiverse might differ. Further, there are discussions concerning the interpretations of Quantum Physics. One interpretation is based on the Copenhagen Program, based on the collapse of the waves of probabilities when measured (observed), and another one belongs to Hugh Everett III ([11]) saying that the probability waves never collapse, but what happens is in fact a bifurcation (multifurcation) of the (rank 2) Multiverse depending on all the possible situations (so, Schrodinger's cat is alive in an Universe and dead in another one, at the same time). This is the so-called many-world interpretation, giving the Tegmark' rank 3 Multiverse (as a quantum family of rank 2 multiverses). And, finally, a rank 4 Multiverse is represented by pure Mathematics, with the Axiom saying that any non-contradictory mathematical theory should have real model(s) in the rank 4 Multiverse (see 5.1.7., 7.1.2., 8.1. also). This tries to explain why our Universe allows life to exist (all the Universal constants should be very, very carefully chosen: the number of Avogadro, the speed of light, the Planck distance or Planck time,...). We will restrict ourselves to a rank 3 Multiverse, as being the quantum family of our observable Universes. While the quantum logic of John von Neumann is considered as being the quantum logic in the Universe (in the sense of the previous definition), the quantum logic in topoi is considered as being the quantum 
logic in the Quantum Multiverse (rank 3). This is the logic which we will use, because it is the most appropriate to the human thinking, which is a product of the brain, a substructure of the Uni(Multi)verse (considered one of the most complex such substructures, along with black holes and dark matter). Coming back to the rank 4 Multiverse, it is based on the postulate that there are physical models for any non-contradictory mathematical theory. However, the best theories from physics, namely the Einstein' Theory of (General) Relativity (modeling the Universe at the macro-level), and the Quantum Physics (modeling the smallest sub-structures on the Uni(Multi)verse, at the nano-, pico-,... level) are in contradiction in some situations (the quantum entanglement is one of them). At the same time, the two theories should coexist in black holes, for instance. So, a new unifying theory is required, that is a theory unifying the Standard Model (which unifies the Electromagnetic, Strong, and Electroweak forces), a quantic model, with the Gravitation (modeled by the General Relativity). There are currently several approaches in this direction: String Theory, Emergent Quantum Mechanics, Loop Gravity,... We will restrict ourselves to some comments relative to the first two of them.

3.2. Infons and Energons in String Theory. In String Theory (see [17]) one considers that any elementary particle is a tiny vibrating chord - string - in a 10-dimensional space, representing the direct product between the Einsteinian 4-dimensional space-time Universe (Einstein-Minkowski real 4-fold) and a complex (projective algebraic) Calabi-Yau 3-fold (so a 6-dimensional real manifold). The Calabi-Yau 3-fold is considered because it has very many symmetries, giving the so-called supersymmetric String Theory, modeling not only bosons (as the initial String Theory did) - that is particles with integer spin, but the fermions as well - particles with half integer but not integer spin. In such a situation the number of elementary particles (quarks, photons, electrons,...) doubled, and one still look for the new elementary particles at CHERN, in Geneve. The 6 additional real dimensions of the Universe (Multiverse) are very compacted, and give only very limited possibilities to the string to vibrate, imposing conditions, as wave lenght, amplitude,... So, a string needs energy, in order to vibrate, and information, in order to vibrate in a specific way to give one or another of the elementary particles (or antiparticles). In [39] one considers hypothetical elementary particles related to information, calling them infons. It is for the first time when the notion has been considered. However, our point of view is based on the fact that these infons represent only one facet of an elementary particle, the other being represented by energons, so an elementary particle is like a coin, with two facets: the infon, and the energon. The theory of infons has been already discussed a little in Section 2. After [39], a theory of infons (propositional calculus) has been built by [18]. They came to the conclusion that this theory is a conservative extension of the intuitionistic logic. However, one can show that the axiomatic of non-standard analysis in an environment using intuitionistic (many-valued) logic represents another similar notion of infon which we will use in this paper. So an infon is given here by a non-standard enlargement of a topos of the form $\mathbf{S E T}^{\mathcal{C}^{o p}}$ (see Section 5.). The Theory of infons is a triplet $\mathcal{I}=(\mathcal{L}$, $\mathcal{A}, \mathcal{D}$ ), where $\mathcal{L}$ is the usual language of the intuitionistic non-standard context (see Section 6.), $\mathcal{A}$ is a set of propositions written in the previous language considered as being true (axioms), and $\mathcal{D}$ is a set of deduction rules (as modus ponens is) - see Section 5. - 7. In our sense, the theory of infons is the theory of non-standard enlargements of the topoi of type SET $^{\mathcal{C}^{o p}}$ (see section 5.). In String Theory, an infon is a non-standard enlargement of a topos of the previous type, while in Emergent Quantum Mechanics an infon remains the theory itself, and the enlargement of the topos of the previous type will be called 
recepton. In String Theory we also need to define the energon. We will do this a little later, but, for now, we remark that :

\subsection{Infons and Receptons in Emergent Quantum Mechanics. In Emergent Quantum Mechanics} [44]) one considers that the (potential) Energy is also Information, namely the information that something can move from one place to another. However, we think that we must distinguish between potential (virtual) energy and actual energy (kinetic, caloric, electromagnetic,...). The matter is considered a condensed form of (actual) energy (under the action of the strong nuclear force) related to energy by the well-known formula $E=m c^{2}$ (both the nuclear fission, from the atomic power plants and the nuclear fusion, from the Sun, generates loss of mass, producing energy). But a stronger argument connecting the (potential) energy with information is represented by The Landauer Principle, quantumising the energy necessary to erase a bit of information (for details see [5]). It means that both energy and information are quantised, and they are somehow inverse proportional (not exponential, as in the case of entropy, see Section 2., but in the same direction, the relation being given by a precise formula, namely $E_{(\min )}=k_{B} T \ln (2)$, where $k_{B}$ is the Boltzmann constant, $T$ is the temperature of the environment and $\ln$ is the natural logarithm. So, in Emergent Quantum Mechnics everything primordially exists as waves of information $=$ theories in a conservative extension of the intuitionistic logic, all of them being infons. Their associated categories (of models) represent then the other facets of the coin, called receptons so, in this setting the two faces are the infon, and the recepton, the category of models of that theory (which is a non-standard enlargement of a topos of a special type, see Section 7.). We, the human beings, are, at the level of our brains, or to the level of the global brain (which include both all the network of the human brains, and the technological discoveries, artificial intelligence included, but also other kind of sensory-type machines) like the radio-sets travelling in a landscape with plenty of electromagnetic waves, and discovering them progressively, function of the increasing quality of the receptor, improved due to the technologies. So, we become progressively aware of the surrounding informational waves (theories) improving progressively our consciousness (both at the individual and global level). At the same time, the subconsciousness works, impacting at every step on the consciousness. So, the receptons become the elementary pseudoparticles of the union between consciousness and subconsciousness, the two facets of the coins being the theory and the associated category (which must be a non-standard topos). In this version of an unification theory, the Uni(QuantumMulti)verse is emerging, being stepwise discovered by brain and global brain through the measurement (collapse) of the intuitionistic informational waves. In fact, Emergent Quantum Mechanics is essentially based on information and consciousness (which becomes part of Physics!), but there are other important facts around, namely the non-determinism (with the meaning that not only the past influences us, but also the future), the discrete space, Gosset Polytope (see Section 5. also, for a topos representing it), E8-crystal, and golden ratio $=\Phi=\lim _{n \rightarrow \infty}\left(F_{n+1} / F_{n}\right)(=(1+\sqrt{5}) / 2)$, where $\left(F_{n}\right)_{n \in \mathbf{N}}$ is the Fibonacci sequence. The $4 \mathrm{D}$ space-time is "pixelated" by the projection on $\mathbf{R}^{4}$ of the Gosset polytope from $\mathbf{R}^{8}$ with an angle which produce on $\mathbf{R}^{4}$ two similar 4-dimensional polytopes such that the quotient of their 4-volumes is equal to $\Phi$. We recall that an Energon is either a theory about a potential work (potential energy), so an Infon, or a real work (actual energy), which is a Recepton. 


\section{Cybernetical Principles of the Global Brain}

In this Section we suggest a notion of the Global Brain, using a Cybernetical approach, relating again Information - Reception - Energy. The approach is both original and based on ideas coming from [27], [28], [45], [9].

4.1. What is the Global Brain? The Global Brain is a bio-technological System composed by physical Receptons organized to generate (electromagnetic, gravitational,...) fields of Energons, fields which interact to the higher levels of Infons and create Consciousness. The Purpose for humans to create a Global Consciousness can be achieved by a smart global architecture of physical Human-Machine System to implement Cybernetic Principles of INFORMATION. As Mihai Draganescu proposed in 1990 there is a frontier between physical level of matter and its Informational dimension called InfoMatter ([9]). At this intersection we identify cybernetic laws which appear in all information systems detected by human senzors (natural and artificial).

\subsection{Cybernetic Principles of Information in the Global Brain:}

1. Autonomy of Information: Information preexists at the highest level of Consciousness and operates individual (finite) and global (infinite).

2. Universal Connectivity of Information manifests on the Matrix of all data connections between and inside physical and non physical phenomena on all dimensions.

3. Free Will versus Information: Human being relates to Information in terms of list of possible actions and non-actions as a basis for Free Will.

4. Authorization Access to In formation: at physical level quantum fields interact to create support for Information, access to Information on macro level of Human Beings consists in permissions for entities as Humans, Robots or other Automatic to interchange data according to Free Will Principle.

5.Exception Handling for Information Ambiguities : Natural phenomena of information error consists of contradictions inside information with associated correction choices (Multiple Choices).

Eric Kandel ([22]) was a recipient of the 2000 Nobel Prize in Physiology or Medicine Nobel Prize for his research on the memory storage in neurons proved that Information is linked inside brain to physical level.

4.3. Global Brain Functions regarding Information are derived from Data Cybernetic Principles ([20]) with the difference Information is a Metadata relevant in terms of utility for a recipient (Human or Robotic).

1.Information aqusition: metadata is identified from perception of existing data inside and outside entities (Humans/Robots, at the Recepton Level).

2.Information processing: metadata is created through algoritmic processing of data perceived through sensors (at the Infon-Energon Level).

3.Information Storage: metadata is physically stored in human brain and IT recipients (natural/artificial neurons and machine storage recipients) (Infon Level).

4.Information sending and receiving: metadata interchange between sender and receiver in Request-Answer process (Recepton Level).

\subsection{Limits and Outbounds of the Global Brain.}

We start with a picture (it is related to picture 7 from [4]): 


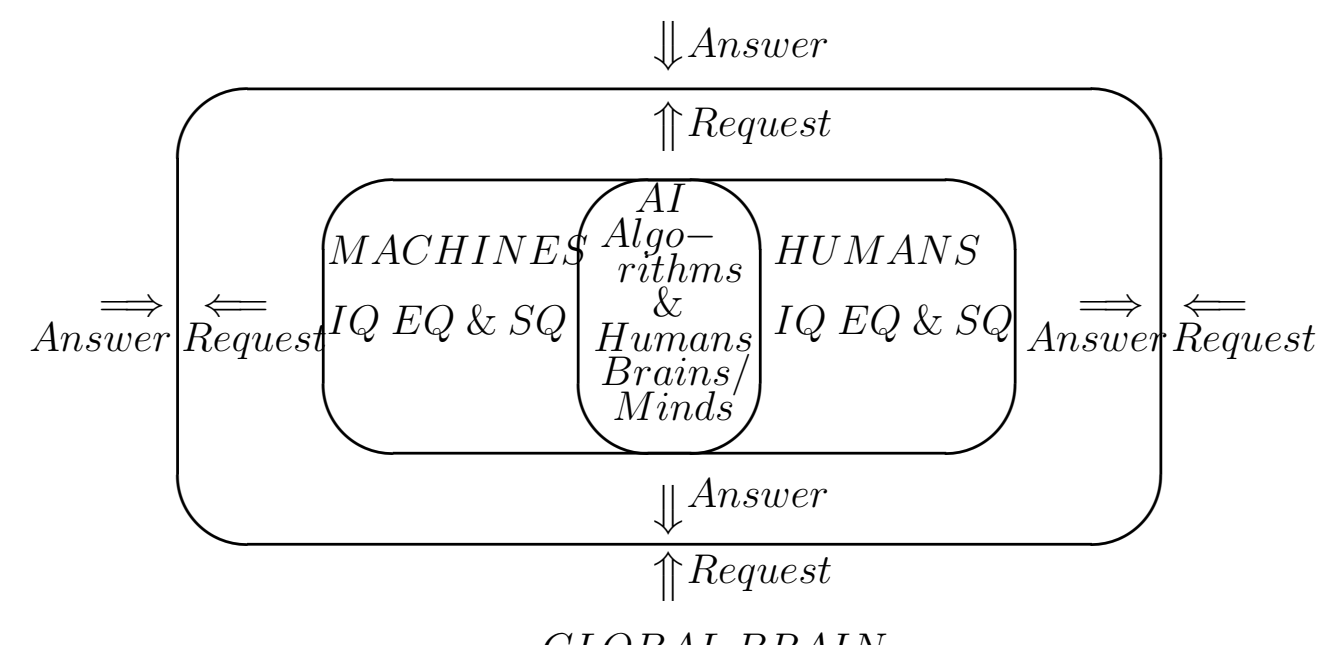

GLOBAL BRAIN

Machine (AI Algorithms) vs. Humans : a Global Brain Architecture

The Architecture of Global Brain generated by Machine (Artificial Intelligence Algorithms) - Human Beings interactions are inspired by Norbert Wiener's Cybernetic Communication principles ([45]), and have a possible graphical representation as below (M-Hemisphere, H-Hemisphere, corpus AIH, analogous to the left hemisphere, right hemisphere and corpus callosum of the human brain). Based on Stefan Odoblejas vision ([27]) on analogy and models of mental psychology, a Global Brain should attain at least the psychological functions of the human brain. Therefore: Request-Answer is a Cybernetic model to create Bio-Technological Feedback between Global Brain entities at the Knowledge Frontier. In the figure above the membrane of the Global Brain is in fact an invisible Knowledge Frontier towards Global Consciousness, continuously in activity inside the System and above it. Many scientists claim for unified theory in science for the explanations of natural phenomena, one of them is Nassim Harramein ([19]) with Unified Field Theory (see also Section 3.), which connects the Standard Model and Gravitation in a single unifying framework (see also [27], [24], [21]). From this viewpoint, the Global Brain is the instrument to apply and study multiple theories from multiple disciplines: psychology, physiology, informatics, cybernetics, physics, mathematics and others in One Brain Framework. Nikola Tesla stated also that we live in the Age of Electricity ([42]) where electricity is the meeting point for chemists, physicians and physiologists, as the electromagnetic energy can be transmitted wireless and its power conducts mankind to progress. In fact Electricity is a basis for any Global System and the Global Brain can be viewed as an application of Tesla's scientific work. Therefore Tesla's vision of communication services in a Global System have become possible to implement almost a hundred years later due to Internet and Machines ([43]). Therefore the image above is a vision of a complex adaptive system composed by artificial Machines able to manifest Rational, Emotional and Social Intelligence (levels of IQ, EQ, SQ) and Humans also given by nature the same characteristics, the communication being done at the Level of Artificial Intelligence based on Cybernetic Algorithms (certainly, the relatively recent discoveries in the aria of gravitational waves can relate the things with the gravitation/graviton also). The final scope of Global Brain is to be a Knowledge instrument used by Humans to develop more self consciousness in their natural habitat. To preserve the habitat of life with other forms of biological and non biological entities, humans should design a smart and self adaptive system with Ethics and Human Psychological Components. Mihai Golu ([15], [16]) studied the field of neuropsychology and its cybernetic dimension and his work is a startpoint to define 
Global Brain to be able to manifest Consciousness at a higher level of performance in comparison to nowadays human society manifestations on all aspects of development.

Regarding Mathematical instrument to design and operate inside Global Brain, the Non Standard Analysis in Topoi is a research startpoint achieved in the following Sections of this work. In this setting, we propose a starting definition for Infons, Energons and Receptons, as working tools used to understand the things better and better. We will formalize in a mathematical language some of the previous comments related to the three pseudoparticles, in accordance with the previous characteristics sketched for them, coming from Biology/Physiology, Physics and Cybernetics. The formalism aims to produce a new mathematical approach for the Problems of Neuroscience, Physics, and Artificial Intelligence. A list with problems from the of Neuroscience is contained in the book [37]. These can be also considered a list of Problem from Physics in Emergent Quantum Mechanics.

\section{Theories versus Categories ; Topoi and Mathematical Logic}

Although the results from this Section are known, we consider this review Section as representing useful preliminaries for the original part of this paper consisting in the construction of the non-standard extensions in topoi of the SET-type, from the next Section 6., and for the original definitions from Section 7. Here we present, in 5.1. the interplay between Theories and Categories in the classical context of the bivalent logic and Zermelo-Fraenkel(Choice) axiomatics, as precursors of the mathematical definitions of the Infons and Receptons from Section 7. Later, in 5.2 and 5.3 we'll pass to a special kind of categories, namely topoi, with their intuitionistic logic detailed in 5.3. For more details concerning the topics from this section, we refer to [7], [33], [36], [13], [34]. See also the extended version [25], which have the same structure as this paper has, but the notions are presented in more detail.

\subsection{Theories versus Categories in $\mathrm{ZF}(\mathrm{C})$ axiomatics as precursors on the Mathematical Infons and Receptons.}

Definition 5.1.1. A class is an arbitrary collection of objects. A set is a class which belongs to another class (one avoid in such a way the well-known Russell's paradox, 5.2 from [25]).

Definition 5.1.2. A language $\mathcal{L}$ is the family of all formulae, obtained inductively using conjunctions, disjunctions, negations, implications and quantification (existential or/and universal) of the atomic formulae (represented by equality and appartenence of variables, sets, t-uples, $\tau(\sigma)$ with $\tau$ and $\sigma$ from before) (see also 5.3 from [25]).

If $\mathcal{N}$ is a family of classes stable to " $\in$ " (that is $\forall y(y \in \mathcal{N} \wedge x \in y \rightarrow x \in \mathcal{N}$ ), then we denote by $\mathcal{L}_{\mathcal{N}}$ the restriction of $\mathcal{L}$ to $\mathcal{N}$ (this means that we replace in the classes with those classes from $\mathcal{N}$ only).

Definition 5.1.3. A sentence (or proposition) is a formula having all the variables bounded (cuantified), so there is no free variable. A sentence is true or false (bivalent logic, see 5.1.9).

Definition 5.1.4. A set of axioms is a set $\mathcal{A}$ of sentences, supposed true.

Definition 5.1.5. The deduction rules are the rules of inferrence from a set $\mathcal{D}$.

Examples 5.1.6.

1. modus ponens (MP): $\frac{p, p \rightarrow q}{q}$; 
2. particularization $(\mathrm{P}): \frac{\forall x p(x)}{p(y)}$;

3. generalization $(\mathrm{G}): \frac{p(y)}{\forall x p(x)}$, etc.

Definition 5.1.7. A theory is a triplet $\mathcal{T}=(\mathcal{L}, \mathcal{A}, \mathcal{D})$, with $\mathcal{L}, \mathcal{A}, \mathcal{D}$ as in 5.1.2, 5.1.4, 5.1.5 respectively; in the classical context $\mathcal{A}$ contains the axioms of Classical Logic (bivalent) $C L$ (see 5.1.8) and the well-known Zermelo-Fraenkel(Choice) $Z F(C)$ axioms. We assume here, by definition, that $a$ theory has at least one model (this represents a compatibility with Tegmark's Rank 4 Multiverse, see Section 3.), which is not automatic for the theories of order at least 2 (see 5.1.13).

Definition 5.1.8. A classical logic (CL) is a theory having the following axioms:

Then $-1: A \rightarrow(B \rightarrow A)$.

Then $-2:(A \rightarrow(B \rightarrow C)) \rightarrow((A \rightarrow B) \rightarrow(A \rightarrow C))$.

And $-1: A \wedge B \rightarrow A$.

And $-2: A \wedge B \rightarrow B$.

And $-3: A \rightarrow(B \rightarrow(A \wedge B))$.

Or $-1: A \rightarrow A \vee B$.

Or $-2: B \rightarrow A \vee B$.

Or $-3:(A \rightarrow C) \rightarrow((B \rightarrow C) \rightarrow(A \vee B \rightarrow C))$.

Not $-1:(A \rightarrow B) \rightarrow((A \rightarrow \neg B) \rightarrow \neg A)$.

Not $-2: A \rightarrow(\neg A \rightarrow B)$ (or $\perp \rightarrow B, \perp$ false).

Not $-3: A \vee \neg A$.

Remark 5.1.9. We recall from $\mathrm{ZF}$ list the following axioms only (see 5.10-5.14 from [25]):

Extensionality (E): $\forall x \forall y[\forall z(z \in x \leftrightarrow z \in y) \rightarrow x=y]$

Regularity (R): $\forall x[\exists a(a \in x) \rightarrow \exists y(y \in x \wedge \neg(\exists z(z \in y \wedge z \in x)))]$

Specification (S): $\forall z \forall \omega_{1} \forall \omega_{2} \ldots \forall \omega_{n} \exists y \forall x[x \in y \leftrightarrow((x \in z) \wedge \varphi)]$, where $\varphi$ is a formula in $\mathcal{L}_{\mathcal{M}}$, with the free variables among $z, \omega_{1}, \ldots, \omega_{n}$ and having $y$ as a bounded variable. In particular there are sets of the form $\{x \mid \varphi(x)\}$; note that this axiom allow us to consider such kind of sets only if they are subsets of a bigger set, avoiding in such a way the Russell' paradox. Moreover, this axiom allow us to built the empty set, specifically

Remark 5.1.10. $\emptyset=\{x \in y \mid x \in x \wedge \neg(x \in x)\}$ (the empty set). Moreover, the empty set is unique (does not depend on $y$ ) by the Axiom $(\mathbf{E})$.

Infinity (I) (Peano): $\exists X[\emptyset \in X \wedge \forall y(y \in X \rightarrow S(y) \in X]$, where $S(\omega):=\omega \cup\{\omega\}$ (the successor of $\omega)$.

Definition-Remark 5.1.11. $\mathbb{N}:=$ the smallest $X$ as before (= the set of natural numbers).

Definition 5.1.12. Let $T$ be a set (possibly $T=\emptyset$ ). Then $\mathcal{U}=\mathcal{U}(T):=\bigcup_{n=0}^{\infty} U_{n}(T)$, where $U_{0}(T)=T$ and $U_{n+1}(T)=U_{n}(T) \cup \mathcal{P}\left(U_{n}(T)\right), \forall n \geq 0$. This is the Universe generated by $T$ (one can consider a bigger Universe, where the union is taken over all the ordinals, by transfinite recurrence - the von Neumann Universe - but we don't need it now). It is constructed from the set $T$, using $Z F$ axioms. We remark here that, even when $T=\emptyset$, we have $0=\emptyset \in U_{0}$, and, if $\omega \in U_{n}$, then $S(\omega)=\omega \cup\{\omega\} \in U_{(n+1)}$, so $\mathbb{N} \subset \mathcal{U}(\emptyset)$. Then, take $T=\mathbb{N}$. We get, using the standard constructions of the number fields, that all of them $(\mathbb{Z}, \mathbb{Q}, \mathbb{R}, \mathbb{C})$ are contained in $\mathcal{U}(\mathbb{N})$, by the definition of $\mathcal{U}(\mathbb{N})$ and the $Z F$ axioms. Continuing, 
it is easy to see that, in fact, all the necessary mathematical structures can be progressively built starting with the empty set $\emptyset$ (whose existence is postulated by $Z F$ ), using the $Z F(C)$ axioms, in higher and higher Universes as before.

Definition 5.1.13. A language $\mathcal{L}_{\mathcal{U}}$, associated with the previous Universe $\mathcal{U}$ can be defined as in 5.1.2. If the quantifiers applies to $U_{0}(T)=T$ only, we call the resulting language, denoted by $\mathcal{L}_{T}$, a first order language, if the quantifiers applies to $U_{1}(T)$ only, we call the resulting language a second order language, denoted by $\mathcal{L}_{U_{1}(T)}, \ldots$,if the quantifiers applies to $U_{n-1}(T)$ only, we call the resulting language a $n$-th order language,..., in this terminology, $\mathcal{L}_{\mathcal{U}}$ being called a higher order language.

Examples of Theories 5.1.14. $\mathcal{T}=(\mathcal{L}, \mathcal{A}, \mathcal{D})$.

1. The theory of sets: $(\mathrm{Set})=(\mathcal{L}, C L+Z F, M P)$;

2. The theory of groups: $\mathcal{G}=(\mathcal{L}, \mathcal{A}=Z F C+C L+1)-4), M P)$, where:

1) $*: G \times G \rightarrow G$, function;

2) $\forall x \forall y \forall z[(x * y) * z=x *(y * z)]$;

3) $\exists e \forall x(x * e=e * x=x)$;

4) $\forall x \exists x^{\prime}\left(x * x^{\prime}=x^{\prime} * x=e\right)$.

Similarly for 3.-... The theory of rings, Theories of fields, of vector spaces, of partially ordered sets,...

5.1.15. We recall that we assumed that all our theories have models (in the case of the first-order language it is known that a theory has a model iff it is not contradictory, that is there is no sentence $p$ such that both $p$ and its negation are deductible.

\section{Examples 5.1.16.}

1. $(\mathbb{Z},+)$ is a model for the theory of groups; $(\mathbb{Z}=$ the set of integers;

2. $(\mathbb{Z},+, \cdot)$ is a model for the theory of rings; and so long.

Definition 5.1.17. The family of all the models (or of a set of models) of a theory, together with the "dictionaries" between them (functions, or other objects, compatible with the structure of the theory) gives a category $\mathcal{C}_{\mathcal{T}}$ associated to the theory $\mathcal{T}$.

\section{Examples 5.1.18.}

1). $\mathbf{G r}$ (category of groups): contains all (or a set of) groups, denoted by $O b(\mathbf{G r})$, and the morphisms between them, that is $\mathrm{Hom}_{G r}(G, H)=\left\{f: G \rightarrow H \mid f\left(x *_{G} y\right)=f(x) *_{H} f(y), \forall x, y \in G\right\}$.

2). Ring (category of rings): $O b$ (Ring) = a class (or set) of rings, $H_{\text {oring }}(R, S)=\{f: R \rightarrow$ $S \mid \forall x \forall y[f(x+y)=f(x)+f(y)] \wedge[f(x \cdot y)=f(x) \cdot f(y)]\}, f\left(1_{R}\right)=1_{S}$. Similarly for fields (the category Field), vector spaces (the category Vect), ordered sets (the category POSET), and so long.

Definition 5.1.19. (Theory of Categories). A category $\mathcal{C}$ is a 4-uple $\left(O b(\mathcal{C}),\left(\operatorname{Hom}_{\mathcal{C}}(A, B)\right)_{A, B \in O b(\mathcal{C})}, \circ,\left(\mathbf{1}_{X}\right)_{X \in O b(\mathcal{C})}\right)$ satisfying the axioms:

(i) $\circ: \operatorname{Hom}_{\mathcal{C}}(A, B) \times \operatorname{Hom}_{\mathcal{C}}(B, C) \longrightarrow \operatorname{Hom}_{\mathcal{C}}(A, C)$

$(f, g) \longmapsto g \circ f$ such that $(h \circ g) \circ f=h \circ(g \circ f) \forall f, g, h$ for which all the compositions are defined;

(ii) $\forall X \forall Y, X, Y \in O b(\mathcal{C}), \forall f \in \operatorname{Hom}_{\mathcal{C}}(X, Y)$, the following diagrams are commutative:

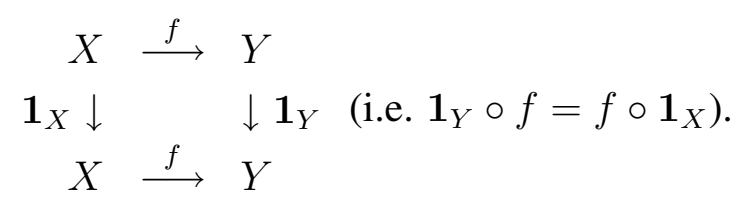


Remark 5.1.20. Any category (the models of a theory) is a model of the theory of categories, as are, for instance, the examples 5.1.16.

Remark 5.1.21. The category associated to the theory of categories is a category of categories, named CAT, that is:

$\mathrm{Ob}(\mathbf{C A T})=$ categories

$\operatorname{Hom}_{\mathbf{C A T}}\left(\mathcal{C}_{1}, \mathcal{C}_{2}\right)=$ the functors $\left(\right.$ see 5.1.22) from $\mathcal{C}_{1}$ to $\mathcal{C}_{2}$, with "o" and $\mathbf{1}_{\mathcal{C}}$ defined canonically.

Definition 5.1.22. Let $\mathcal{C}_{1}$ and $\mathcal{C}_{2}$ be two categories. A functor $F: \mathcal{C}_{1} \rightarrow \mathcal{C}_{2}$ is acting on objects: $F(A) \in O b\left(\mathcal{C}_{2}\right), \forall A \in O b\left(\mathcal{C}_{1}\right)$ and on morphisms: $F(f) \in \operatorname{Hom}_{\mathcal{C}_{2}}(F(X), F(Y)), \forall f \in$ $H_{o m} \mathcal{C}_{1}(X, Y)$, being compatible with compositions and identities, that is:

(i) $F(g \circ f)=F(g) \circ F(f), \forall f, g$ such that the composition is defined, and

(ii) $F\left(\mathbf{1}_{X}\right)=\mathbf{1}_{F(X)}, \forall X \in O b\left(\mathcal{C}_{1}\right)$.

Remark 5.1.23. The previous definition introduce both the notion of morphism in CAT, and the theory of functors also. So, one expects to get the Category of functors also. Indeed,

Definition 5.1.24. Let $\mathcal{C}_{1}$ and $\mathcal{C}_{2}$ be two categories. We define the category $\mathcal{C}_{2}^{\mathcal{C}_{1}}$ as follows:

- $O b\left(\mathcal{C}_{2}^{\mathcal{C}_{1}}\right)=\left\{F: \mathcal{C}_{1} \rightarrow \mathcal{C}_{2} \mid F\right.$ functor $\}$

- $\operatorname{Hom}_{\mathcal{C}_{2}^{\mathcal{C}_{1}}}\left(F_{1}, F_{2}\right)=\left\{h: F_{1} \rightarrow F_{2} \mid h\right.$ is a natural transformation $\}$.

The composition and the identities are defined canonically.

Here we need the following:

Definition 5.1.25. If $F_{1}, F_{2} \in O b\left(\mathcal{C}_{2}^{\mathcal{C}_{1}}\right)$, a natural transformation $h: F_{1} \rightarrow F_{2}$ is a collection $h=\left(h_{X}\right)_{X \in O b\left(\mathcal{C}_{1}\right)}$ of morphisms $h_{X}: F_{1}(X) \rightarrow F_{2}(X), h_{X} \in H_{o m}{\mathcal{C}_{2}}_{2}\left(F_{1}(X), F_{2}(X)\right), \forall X \in O b\left(\mathcal{C}_{1}\right)$ $F_{1}(X) \stackrel{h_{X}}{\longrightarrow} F_{2}(X)$

such that $\forall f \in \operatorname{Hom}_{\mathcal{C}_{1}}(X, Y)$ the diagrams $F_{1}(f) \downarrow \quad \downarrow F_{2}(f)$ commutes (i.e. $h_{Y} \circ F_{1}(f)=$ $\left.F_{2}(f) \circ h_{X}\right)$.$$
F_{1}(Y) \stackrel{h_{Y}}{\longrightarrow} F_{2}(Y)
$$

Remark 5.1.26. We insisted till now to get categories from theories, but the converse also works, axiomatizing the things. So, the theories and the categories are the two faces of the same thing, but they represent different notions.

Definition 5.1.27. Let $\mathcal{C}$ be a category. The dual category of $\mathcal{C}$, denoted by $\mathcal{C}^{o p}$ is the category having:

- $O b\left(\mathcal{C}^{o p}\right)=O b(\mathcal{C})$;

- $\operatorname{Hom}_{\mathcal{C}^{o p}}(X, Y)=\operatorname{Hom}_{\mathcal{C}}(Y, X), \forall X, Y \in O b(\mathcal{C})$ (reversing the arrows).

5.1.28. We will assume further that the reader is familiar with parts of the Theory of Categories. If not, we produced the manuscript [25] (Sections 5. and 6.) exactly for those readers (from Neuroscience, or some Physicists, Economists) which feel better to have all the necessary things collected in only one place. A much more detailed treatment is presented in [13]. In particular, we assume as known the notions of initial object (usually denoted by $\mathbf{0}$ ), final object (usually denoted by 1), monomorphism, epimorphism, isomorphism, subobject of an object, direct product, direct sum, equalizer, coequalizer, inductive limit, projective limit, pullbacks, pushouts, exponentiation (usually denoted by $\mathbf{X}^{\mathbf{Y}}$, with $\mathbf{X}, \mathbf{Y}$ objects), subobjects classifier (usually denoted by $\Omega$ ). The notions product and sum, initial and final, epi and mono, co... and without co, pull and push, inductive and projective,... are dual notions. An example is given by the next 
Definition 5.1.29. A functor $F: \mathcal{C}_{1} \rightarrow \mathcal{C}_{2}^{o p}$ is called a cofunctor $F: \mathcal{C}_{1} \rightarrow \mathcal{C}_{2}$. It satisfies $F(g \circ f)=F(f) \circ F(g), \forall f, g$ (instead of $F(g \circ f)=F(g) \circ F(f), \forall f, g$ as a functor does). This is the first example of a pair of dual notions (functor vs. cofunctor). We'll see other examples in section 6.

5.2. Topos Theory. Now we pass to the main notions used in this paper:

Definition 5.2.1. An elementary topos is a category $\mathcal{E}$ such that:

(T1) $\mathcal{E}$ has finite inductive limits;

(T2) $\mathcal{E}$ has finite projective limits;

(T3) $\mathcal{E}$ has exponentiation;

(T4) $\mathcal{E}$ has subobjects classifier. (see [13], [25])

Remark 5.2.2. It can be shown that the conditions required in the definition of a topos, implies the existence in such categories of the objects having the propriety to be any of the objects from 5.1.28. (so, there exists initial and final objects, (finite) direct products and sums, equalizer, coequalizer, pullbacks, pushouts, power object of any two objects,...)

Examples of topoi 5.2.3. 1). The category SET of sets.

2 ). The category of $n$-uples of sets with the morphisms defined on components. This can be written as $\mathbf{S E T}^{\mathbf{n}}$, where $\mathbf{n}$ is the discrete category having $O b(\mathbf{n})=\{1,2, \ldots, n\}, 1,2, \ldots \in \mathbb{N}$ as objects and only the identities as morphisms (this category is of type 5.1.24).

3). SET $^{\mathcal{C o p}}$ is also a topos. The previous example is a particular case, when $\mathcal{C}=\mathbf{n}$.

Definition 5.2.4. A topos of the form 3). will be called exponential topos. If the topos from 3 ) is obtained for a category $\mathcal{C}$ having a finite number of objects and morphisms, we will call the topos SET-type topos.

Remark 5.2.5. In Section 6. we will construct non-standard extensions for SET-type topoi, as finite-generalization of the non-standard analysis in the topos SET, introduced in [35], see also [14], [25]. This will be an original mathematical contribution. The general case of the exponential topos will be considered in Part II of this work.

5.2.6. Oriented graphs as topoi of SET-type. An oriented graph $\overrightarrow{\mathcal{G}}$ consists in a finite number of points $A=\left\{A_{1}, A_{2}, \ldots, A_{m}\right\}$ (the word point, is only formally used,the elements of A are not necessarily geometric entities), some (or all) of them being joined with some others (or all) of them by oriented segments (arrows); we note that the elements of $A$ can be categories, and the arrows can be functors (for example). We will suppose here, additionally, that for each $i, j, i, j \in\{1, \ldots, n\}$ there is at most one arrow, either from $A_{i}$ to $A_{j}$ or from $A_{j}$ to $A_{i}$, that the path of two consecutive arrows can be replaced with an arrow, and we implicitely assume the existence of an unique arrow from $A_{i}$ to itself, for any $i$ as before, namely the identity arrow (which has no orientation). We can organize this graph as a category of type $\mathbf{n}$ with some aditional morphisms: we add to $\mathbf{n}$ a morphism from $i$ to $j$, $i, j \in \mathbf{n}$ iff there is an arrow from $A_{j}$ to $A_{i}$ in $\overrightarrow{\mathcal{G}}$. Let's denote this new category by $\mathbf{n}_{\overrightarrow{\mathcal{G}}}$. Then the SET-type topos $\mathbf{S E T}^{\mathbf{n}_{\overrightarrow{\mathcal{G}}}{ }^{o p}}$ can replace the given graph. In fact one can work with a category of n-uples of sets, $\left(X_{1}, \ldots, X_{n}\right)$ requesting additionally that $X_{i} \subset X_{j}$ whenever there is an arrow from $A_{i}$ to $A_{j}$ in $\overrightarrow{\mathcal{G}}$. This approach can be used to study various kind of graphs, in particular graph neural (quantum) networks of various kinds (optical, convolutional,...) with the quantum aspects modeled in topoi. Such a development is related to deep learning, artificial intelligence, quantum computers (starting with the 
quantum Turing mashine). Moreover, Mathematical Game Theory is also related to Graph Theory, and some economical applications can be obtained, for instance related to the new aria of Neuroeconomics. There are also connections between graphs and the evolutionary games (with applications in the study of climate change, evolutionary biology,...).

5.2.7. Geometric topoi. One can use the previous topos-interpretation of the oriented graphs to build Geometric Topoi. Let's consider the following oriented tetrahedron :

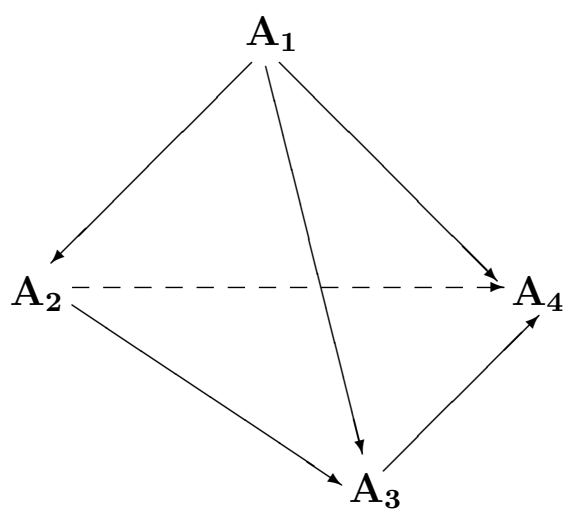

We denote now by $\overrightarrow{\mathcal{T}}$ the oriented graph given by the previous tetrahedron, and by $\mathbf{n}_{\overrightarrow{\mathcal{T}}}$ the discrete category $\mathbf{n}$ at which we added the additional morphisms $2 \rightarrow 1,3 \rightarrow 1,4 \rightarrow 1,3 \rightarrow 2,4 \rightarrow 2,4 \rightarrow 3$. Then the previous tetrahedron can be recognized in the topos $\mathbf{S E T}_{\overrightarrow{\mathcal{T}}}^{\text {op }}$ or, alternatively, in the topos whose objects are the quadruplets $\left\{\left(A_{1}, A_{2}, A_{3}, A_{4}\right) \mid A_{1}, A_{2}, A_{3}, A_{4}\right.$ sets, with $A_{1} \subset A_{2}, A_{1} \subset A_{3}, A_{1} \subset$ $\left.A_{4}, A_{2} \subset A_{3}, A_{2} \subset A_{4}, A_{3} \subset A_{4}\right\}$ and having as morphisms quadruplets of functions compatible with restrictions. In such a way, one can represents in topoi any polygon, polyhedron, and polytope from $\mathbf{R}^{n}$, $n \geq 2$.

5.2.8. Gosset polytope. An important polytope in Emergent Quantum Mechanics (where the consciousness become part of Physics) is the Gosset polytope (see the final part of section 4.). It is a polytope $P \subset \mathbf{R}^{8}$ having 240 vertices, with coordinates $( \pm 2, \pm 2,0,0,0,0,0,0)$, with any combination of signs and the 2's on any two positions (112 vertices) and ( $\pm 1, \pm 1, \pm 1, \pm 1, \pm 1, \pm 1, \pm 1, \pm 1$ ), by taking an even number of minus signs (128 more vertices). The vertices of the analogue of the Gosset polytope in $\mathbf{R}^{3}$ has the coordinates $( \pm 2, \pm 2,0),( \pm 2,0, \pm 2),(0, \pm 2, \pm 2)$ (12 vertices), and $(-1,1,1),(1,-1,1),(1,1,-1),(-1,-1,-1)$ so, this polyhedron has 16 vertices. They form 3 squares of radious 4 , with the centers in the center of the axes of coordinates and sitting in the planes $\mathrm{xOy}, \mathrm{yOz}$, $\mathrm{zOx}$ respectively ( $\mathrm{O}$ is the center of the axes), and having the edges parallel with the axes of coordinates, and a regular tetrahedron. The 12 vertices of the three squares are the middle of the edges of a cube having edges of length 4 , the center in $\mathrm{O}$, and the faces paralel with the planes of coordinates. So, this polyhedron is determined by a cube and a (regular) tetrahedrun. It can be represented as a topos using the method from 5.2.7. But even the Gosset polytope from $\mathbf{R}^{8}$ can be represented in such a way once we give orientations to its edges. However, the group of symmetries of the Gosset polytope is a group which can be found in the structure of the (-2) curves from a degenerate del Pezzo surface of degree $d$. However, we will deal with this subject in furthcoming paper, based on [29], [30]. 
5.3. Intuitionistic Logic in Topoi. It is well known the connection between CL (see 5.1.8.) and the Boolean Algebras (resp. lattices), $\mathcal{A}=(A,+, \cdot,-, 0,1)$ (resp. $=\left(A, \vee, \wedge,{ }^{\prime}, 0,1\right)$, with the operations defined canonically: sup, inf,...), see [36] ([25] might be also useful). We'll not insist here.

Definition 5.3.1. The axioms of (IL) are (notations from 5.1.8.):

Then - 1, Then - 2;

And - 1, And - 2, And - 3;

Or - 1, Or - 2, Or - 3;

Not 2.

Definition 5.3.2. A poset $(H, \leq)$ is called a Heyting lattice if it is a bounded lattice (so, for any $a, b \in H$ there exists $a \wedge b=\inf \{a, b\}, a \vee b=\sup \{a, b\}$, there exists $0,1 \in H$ such that $0 \leq x \leq 1$ for any $x \in H$ ) satisfying the following conditions a) - e), when replacing + and $\cdot$ by $\vee$ and $\wedge(0,1$ remain the same), and having an operation $\rightarrow$ satisfying:

$$
\forall a, b, c,(a \wedge b \leq c \Leftrightarrow a \leq(b \rightarrow c))
$$

Here: a) $\forall x \forall y(x+y=y+x) ; \forall x \forall y:(x \cdot y=y \cdot x)$;

b) $\forall x \forall y \forall z[(x+y)+z=x+(y+z)] ; \forall x \forall y \forall z[(x \cdot y) \cdot z=x \cdot(y \cdot z)]$;

c) $\forall x \forall y \forall z[x \cdot(y+z)=x \cdot y+x \cdot z] ; \forall x \forall y \forall z[x+(y \cdot z)=(x+y) \cdot(x+z)]$;

d) $\forall n(x+0=0+x=x) ; \forall n(x \cdot 0=0 \cdot x=0)$;

e) $\forall n(x+1=1+x=1) ; \forall n(x \cdot 1=1 \cdot x=x)$;

So, a Heyting lattice is a 6 -uple $(H, \vee, \wedge, \rightarrow, 0,1)$ as before. A Heyting algebra is a 6-uple $(H,+, \cdot \rightarrow$ $, 0,1)$ obtained by replacing $\vee$ and $\wedge$ with + and $\cdot$. Given a Heyting algebra we get from it a poset which is a Heyting lattice, putting $a \leq b \Leftrightarrow a \rightarrow b=1$.

A monadic Heyting algebra (abbreviated MHA) is an algebraic structure $(H,+, \cdot, \rightarrow, 0,1, \exists, \forall)$ where $(H,+, \cdot, \rightarrow, 0,1)$ is a Heyting algebra, $\exists$ is a closure operator on $H$ (this means, it satisfies the following (i)-(iv)), $\forall$ is an interior operator on $H$ (this means, it satisfies the following (i)' ${ }^{\prime}$-(iv)' ${ }^{\prime}$ ), and

(v) $\forall \exists a=\exists a$, for any $a \in H$;

(vi) $\exists \forall a=\forall a$, for any $a \in H$;

(vii) $\exists(\exists a \wedge b)=\exists a \wedge \exists b$, for any $a, b \in H$, where

(i) $\exists 0=0$;

(ii) $a \leq \exists a$, for any $a \in B$ (recall that $a \leq b$ iff $a \cdot b=a$, iff $a+b=b$, iff $a \rightarrow b=1$; we recall that $a \rightarrow b=\bar{a}+b)$

(iii) $\exists a=\exists \exists a$, for any $a \in B$;

(iv) $\exists(a \vee b)=(\exists a) \vee(\exists b)$, for any $a, b \in B$, and

(i) $\forall 1=1$;

(ii) $\forall a \leq a$, for any $a \in B$;

(iii) ${ }^{\prime} \forall a=\forall \forall a$, for any $a \in B$;

(iv) $)^{\prime} \forall(a \wedge b)=(\forall a) \wedge(\forall b)$, for any $a, b \in B$.

Note. Here $a \wedge b=a \cdot b, a \vee b=a+b$ (we preferred to use the logic related symbols).

Remark 5.3.3. The condition (1) from 5.3.2. is satisfied in the Boolean algebra case for $b \rightarrow c=b^{\prime} \vee c$. If, in the definition of MHA we have $\mathrm{H}=\mathrm{B}=$ Boolean algebra, then we use the notation MBA (monadic 
Boolean algebra). In this case, the existence of the closure operator is equivalent to the existence of the interior operator, and the conditions (v) - (vii) from before are automatically satisfied.

Remark 5.3.4. Any Heyting algebra (lattice) satisfies the axioms of (IL) from 5.2.1.

Examples 5.3.5. 1). All the Boolean algebras (lattices) are Heyting algebras (lattices). Any MBA is MHA.

2). An example of a Heyting algebra which is not Boole can be found in [25], at 6.5. 2).

3). Another example of Heyting non-Boole algebra is : If $(X, \tau)$ is a topological space. If $A \subset X$, we define the interior of $A$ as being the set $\left\{a \in A \mid \exists D_{a} \in \tau\right.$ s.t. $\left.a \in D_{a} \subset A\right\}:=\stackrel{\circ}{A}$. Then $(X, \vee, \wedge, \rightarrow 0,1)$ is a Heyting algebra which is not Boolean in general, where: for $A, B \in \tau, A \vee B:=A \cup B \in \tau$, $A \wedge B:=A \cap B \in \tau, A \rightarrow B:=\widehat{\circ} \overline{B \backslash A} \in \tau, 0=\phi \in \tau, 1=X \in \tau$.

Definition 5.3.6. We can define a negation in Heyting algebra, putting $\neg a:=a \rightarrow 0$ (this is compatible with the Boolean case), but it does not satisfy the principle of double negation and other principles (tertium non datur, reductio ad absurdum, De Morgan's laws).

Remark 5.3.7. Given a topos $\mathcal{E}$, one can define, for any $X \in O b(\mathcal{E})$, operations on its subobjects, similar in some sense with the union, intersection, complementary, empty set, total set from the case SET. In a general topos, for any $a, b \in S u b_{\mathcal{E}(X)}$ (Sub = the family of subobjects) one defines: union $a \cup b$ (using the existence of the equalizers and direct sums), intersection $a \cap b$ (using the existence of pullbacks), implication $a \rightarrow b$ (using the existence of the exponentiations), minimal object $=0 \cap X$, and maximal object $=1 \cap X$. In the case of SET, the usual operations between sets gives a structure of Boolean algebra. In a general topos, the previous operations gives a structure of a Heyting algebra. We get an analogue of the power set of X. We suppose all these things as known. For details, see [13], [34] (the constructions are included in 6.37 - 6.42 from [25] also).

Remark 5.3.8. For the topos SET, the subobjects classifier is $\Omega=\{0,1\}$ (via the usual characteristic function), and, in $\mathrm{CL}$ and $\mathrm{ZF}(\mathrm{C})$ axiomatics, the number of truth values is the cardinal of $\operatorname{Hom}_{\mathbf{S E T}}(\mathbf{1}, \Omega)$ (here $\mathbf{1}=$ any singleton), that is 2 . For a general topos, on $\Omega$ one can define logic operators $\vee, \wedge, \rightarrow, \perp, \top$ using the so called $\Omega$-axiom appearing in the definition of the subobjects classifier. Then $(\Omega, \vee, \wedge, \rightarrow, \perp, \top)$ is a Heyting algebra, in general. When it is a Boole algebra, one says that the topos $\mathcal{E}$ is a Boolean topos. We again assume these things as known. Details, again in [13], [34] (the constructions are also included in 6.43, 6.44 from [25]). Moreover, one can give to $\Omega$ a structure of MHA also. See [6], [40] (referred in [13], pag. 457). See also 6.51 - 6.53 from [25].

Remark 5.3.9. Fix a language $\mathcal{L}$, and, for a given topos $\mathcal{E}$ having $\Omega$ as subobjects classifier, we assign to any atomic sentence $a$ from $\mathcal{L}$ (see 5.1.2, 5.1.3) an element $\mathbf{V}(a)$ from $\operatorname{Hom}_{\mathcal{E}}(\mathbf{1}, \Omega)$. Then extend this association $\mathbf{V}$ to all the sentences from $\mathcal{L}$, using the logical connectiveness for $\mathcal{L}$, and, correspondingly, the operators from 5.3.8. in $\operatorname{Hom}_{\mathcal{E}}(\mathbf{1}, \Omega)(\mathbf{V}(p \vee q)=\mathbf{V}(p) \vee \mathbf{v}(q)$, with the $\vee$ from the left being the logical connector, and $\vee$ from the right being the connector from 5.3.8., similarly for the other operations $\wedge$, etc.). This extends the known Boolean valuations. We remark that $\operatorname{card}\left(\operatorname{Hom}_{\mathcal{E}}(\mathbf{1}, \Omega)\right)$ represents the number of truh values in $\mathcal{E}$ (possible infinite). They are called the global sections of $\Omega$.

Example 5.3.10. Consider the SET-type topos $\mathcal{E}$, whose objects are $\left\{\left(A_{1}, A_{2}, A_{3}, A_{4}\right) \mid A_{1} \subset\right.$ $A_{2}, A_{3} \subset A_{4}, A_{1} \subset A_{3}, A_{2} \subset A_{4}$, with morphisms quadruplets of functions, compatible with the existing inclusions. In this cases $\operatorname{card}\left(\operatorname{Hom}_{\mathbf{S E T}_{2}^{4}}(1, \Omega)\right)=6$, the sections being $\{(0,0,0,0)<$ $(0,0,0,1)<(0,0,1,1)<(0,1,0,1)<(0,1,1,1)<(1,1,1,1)\}$ (lexicographical order); the 
characteristic function of the quadruplet of sets is the corresponding quadruplet of usual characteristic functions (for sets). So the intuitionistic logic in this topos has 6 truth values. In such a way we can built as many examples as we want. In particular we can prove that, for any natural number $\mathrm{n}$, there is a topos whose intuitionistic logic has exactly $\mathrm{n}$ truth values. One example of such a topos is the topos having as objects the n-uples of sets $\left\{\left(A_{1}, \ldots, A_{n}\right) \mid A_{1} \in A_{2} \in \ldots A_{n}\right\}$ and as morphisms n-uples of functions, compatible with the inclusions. The the truth values are $\{(0, \ldots, 0)<(0, \ldots, 0,1)<(0, \ldots, 0,1,1)<$ $\ldots<(1, \ldots, 1)\}$, so $(n+1)$ truth values. For more details and examples, see [13], [34] (see also 5.46 5.52 and $6.45-6.53$ from [25]).

\section{Non-standard Analysis in SET-Type Topoi}

We start this Section be shortly recalling (in 6.1) the notion of non-standard enlargement of a standard universe (see 5.1.12) $\mathcal{U}=\mathcal{U}(\mathrm{T})$ (with its mathematics), $\mathcal{U} \subset{ }^{*} \mathcal{U}$, as introduced by Abraham Robinson in [35]. This works in the category (topos) SET. Modern details can be found in [14]. See also [32], [31]. Most of the results from [14] are also presented in 7.20 - 7.45 from Section 7. of [25]. Then, in 6.2, we extend the things to SET-type topoi, introducing for the first time non-standard extensions for such kind of topoi. These are original results from pure mathematical point of view.

6.1. The Non-standard extensions in SET. Let $\mathcal{U}=\mathcal{U}(T) \stackrel{*}{\hookrightarrow} \mathcal{U}^{\prime}=\mathcal{U}\left(T^{\prime}\right)\left(*(T) \subset T^{\prime}\right)$ a monomorphism (injective map) of universes. Let's denote by ${ }^{*} \mathcal{U}:=*(\mathcal{U}) \subseteq \mathcal{U}^{\prime}$ and by ${ }^{*} a$ the image of $a,{ }^{*} a:=*(a), \forall a \in \mathcal{U}$. For a sentence $\varphi$ in $\mathcal{L}_{\mathcal{U}}$, let's denote by ${ }^{*} \varphi$ the sentence in $\mathcal{L}_{\mathcal{U}^{\prime}}$ obtained by keeping unchanged the primary relations $\in$ and $=$ as well as the logical quantifiers $\vee, \wedge, \neg, \rightarrow, \forall, \exists$, and replacing all the constant symbols $a \in \mathcal{U}$ by ${ }^{*} a \in \mathcal{U}^{\prime}$. One requests that $*$ satisfies the axioms:

- * $a=a, \forall a \in T$ (if $\mathcal{U}=\mathcal{U}(T)$, for $T=\emptyset$ this condition doesn't exist);

- $\emptyset=\emptyset$;

- $S, T:$ An $\mathcal{L}_{\mathcal{U}}$ - sentence $\varphi$ is true $\Leftrightarrow{ }^{*} \varphi$ is true as $\mathcal{L}_{\mathcal{U}^{\prime}}$ - sentence; the $\Rightarrow$ is called transfer $(\mathbf{T})$, and $\Leftarrow$ is called standardization $(\mathbf{S})$;

- $I$ (idealisation): If $\mathcal{R} \in \mathcal{U}$ is any finitely satisfiable binary relation (i.e. $\forall n \forall x_{1}, \ldots, x_{n} \exists y\left(y R x_{1} \wedge\right.$ $\left.\ldots \wedge y R x_{n}\right)$ ), then ${ }^{*} \mathcal{R}$ is globally satisfiable (i.e. $\exists y^{\prime} \forall x\left(y^{*} \mathcal{R} x\right)$ ). We should remark that the $x$ 's and $y$ run inside $\mathcal{U}$, while $y^{\prime}$ runs through $\mathcal{U}^{\prime}\left({ }^{*} x=x, \forall x \in \operatorname{dom} \mathcal{R}, y \in \mathcal{U}, y^{\prime} \in \mathcal{U}^{\prime}\right)$. We note that the order of the variables is not important, because, if $\mathcal{R}=\mathcal{R}(x, y)$ is a binary relation, so is $\mathcal{R}(y, x)=\mathcal{R}^{-1}$.

In the previous situation, one call the extension $\mathcal{U} \stackrel{*}{\hookrightarrow} \mathcal{U}^{\prime}$, a non-standard extension of $\mathcal{U}$. We note that the inclusion $\mathcal{U}(T) \subset \mathcal{U}\left({ }^{*} T\right),\left({ }^{*} T \in \mathcal{U}^{\prime}\right)$ is a non-standard extension ([14], Enlargement Theorem, page 185).

Remark 6.1.1. 1 . If $\mathcal{U}=\mathcal{U}(T)$ and $\mathcal{U}^{\prime}=\mathcal{U}\left(T^{\prime}\right)$ are Universes, with $T \subset T^{\prime}$, then take $T^{\prime \prime}$ such that $T \subset T^{\prime \prime}, T^{\prime} \subset T^{\prime \prime}$ and let $\mathcal{U}^{\prime \prime}=\mathcal{U}\left(T^{\prime \prime}\right)$. Then $\mathcal{L}:=\mathcal{L}_{\mathcal{U}}=\mathcal{L}_{\mathcal{U}^{\prime \prime}} \mid \mathcal{U}$ and $\mathcal{L}^{\prime}:=\mathcal{L}_{\mathcal{U}^{\prime}}=\mathcal{L}_{\mathcal{U}^{\prime \prime}} \mid \mathcal{U}^{\prime}$ (notations from 5.1.13). We call ZFC $+\mathrm{CL}+\mathrm{I}+\mathrm{S}+\mathrm{T}:=\mathrm{IST}$ (internal set theory), with $\mathrm{CL}$ from 5.1.8., ZFC from 5.1.9., and I,S,T from before. The triplet $\left(\mathcal{L}=\left(\mathcal{L}_{\mathcal{U}}, \mathcal{L}_{\mathcal{U}^{\prime}}\right), I S T, \mathcal{D}=\{M P, G, P\}\right)$ (see 5.1.6.) represents the Theory of non-standard extensions. We point out that this theory has a higher order logic, that is, it is possible to quantify over any entity. 
2. If $\mathcal{U}$ has ZFC then ${ }^{*} \mathcal{U}$ has IST as axiomatics. Moreover, a sentence $\varphi$ in $\mathcal{L}_{\mathcal{U}}$ conserves its truth value ( 0 or 1 ) when passing to ${ }^{*} \varphi$ in $* \mathcal{U}$. We say that the extension $\mathcal{U} \stackrel{*}{\hookrightarrow} * \mathcal{U}$ is conservative. Moreover, $\mathcal{U}$ is not contradictory iff $* \mathcal{U}$ is (because we can construct models for ${ }^{*} \mathcal{U}$ starting from $\mathcal{U}$ with $Z F C$, see [14], or look at [25] $7.38-7.40$ ).

Remark 6.1.2. One can define the sets ${ }^{*} \mathbb{N},{ }^{*} \mathbb{Z},{ }^{*} \mathbb{Q},{ }^{*} \mathbb{R},{ }^{*} \mathbb{C}$ of the non-standard number sets. In particular, in ${ }^{*} \mathbb{R}$ one gets the notions of infinitezimal, unlimited, infinite etc. numbers. We also can get further proprieties of non-standard numbers. See [14], or look at 7.28 from [25].

6.1.3. Some properties of $*$ (for more of them, see [14], or look at 7.29 from [25]) :

- $a=b$ iff ${ }^{*} a={ }^{*} b$ so $a \mapsto{ }^{*} a$ is injective;

- $a \in B$ iff * $a \in *^{*} B$;

- $A \subseteq B$ iff * $A \subset{ }^{*} B$;

- $\left(a_{1}, \ldots, a_{m}\right)=\left({ }^{*} a_{1}, \ldots,{ }^{*} a_{m}\right), \forall m$;

- $*\left\{a_{1}, \ldots, a_{m}\right\}=\left\{* a_{1}, \ldots, * a_{m}\right\}, \forall m$.

Definition 6.1.4. A set $B \in \mathcal{U}^{\prime}$ is called standard if $\exists A \in \mathcal{U}$ with $B={ }^{*} A$, otherwise it is called non-standard. A non-standard set $B \in{ }^{*} \mathcal{U}$ is called internal if $\exists A \in \mathcal{U}$ with $B \in{ }^{*} A$ and external otherwise.

6.1.5. Some Properties of internal sets. (for more proprieties, examples and further developments, see [14], or look at $7.31-7.45$ from [25]):

- $A, B$ internal $\Rightarrow A \cap B, A \cup B, A \backslash B, A \times B$ internal;

- the union and intersection of any internal collection of sets are internal: if $\left\{A_{i} \mid i \in I\right\} \in{ }^{*} \mathcal{U}$ and $A_{i}$ internal for any $i$, then $\bigcup_{i \in I} A_{i}$ and $\bigcap_{i \in I} A_{i}$ are internal;

$\bullet$ if $a_{1}, \ldots, a_{m}$ are internal, then both $\left\{a_{1}, \ldots, a_{m}\right\}$ and $\left(a_{1}, \ldots, a_{m}\right)$ are internal;

- if $f: A \rightarrow B$ is internal, $a \in \operatorname{dom} f=A$ internal, then $f(a)$ is internal; $C \subseteq A$ internal, then $f^{i}(C)$ is internal; $D \subset B$ internal, then $f^{-1}(D)$ is internal.

Example 6.1.6.

1. $\mathbf{N}$ is an external set in $\mathcal{U}^{\prime}$.

2. ${ }^{*}(A):=\left\{{ }^{*} a \mid a \in A\right\}, A$ infinite is external (note that ${ }^{*}(A) \neq{ }^{*} A$ ).

6.2. Non-standard Analysis in SET-type topoi. We start now to achieve our main objective from the mathematical point of view, namely to build a Non-standard Analysis (NSA) in topoi, extending the previous theory from the classical setting (in the topos SET) to more general topoi. We will be only interested in building NSA in the exponential topoi of the form SET $^{\mathcal{C}^{o p}}$ (such kind of topoi were also used in Quantum Physics). However, for the moment we will give the general definitions, but we will exemplify the ideas on the SET-type topoi (see 5.2.4.).

Remark 6.2.1. Let $\mathcal{E}$ be a topos. In 5.3.8. we recalled the existence of all the logical operations in the space of sections $\operatorname{Hom}_{\mathcal{E}}(1, \Omega)$, such that this space become a $M H A$ (see 5.3.2.). Hence, we can interpret the notion of theory (in the sense of the 5.1.7.) in any topos.

Definition 6.2.2. Let $\mathcal{E}$ be a topos, and $X \in O b(\mathcal{E})$. Then we can associate to any two morphisms $\alpha \in \operatorname{Hom}_{\mathcal{E}}(1, X)$ and $\beta \in \operatorname{Hom}_{\mathcal{E}}(1, X)$ their direct product $(\alpha, \beta) \in \operatorname{Hom}_{\mathcal{E}}(1, X \times X)$ (1 is (the) final object, so $1 \times 1=1)$. Then, a binary relation $\mathcal{R}$ on $\mathrm{X}$ is a set of pairs $(\alpha, \beta)$ as before. As usually, we 
write $\alpha \mathcal{R} \beta$ instead of $(\alpha, \beta) \in \mathcal{R}$. We say that a binary relation on $\mathrm{X}$ in finitely satisfiable iff for any positive integer $n$ and $\alpha_{1}, \ldots, \alpha_{n} \in \operatorname{Hom}_{\mathcal{E}}(1, X)$ there is $\beta \in H o m_{\mathcal{E}}(1, X)$ such that $\alpha_{1} R \beta, \ldots, \alpha_{n} R \beta$.

Definition 6.2.3. Let $\mathcal{E}$ be a topos. An enlargement of $\mathcal{E}$ is a monomorphism ${ }^{*}: \mathcal{E} \hookrightarrow^{*} \mathcal{E}$ in a category CAT containing both categories $\mathcal{E}$ and ${ }^{*} \mathcal{E}$, satisfying :

- 1. * $\mathcal{E}$ is a topos;

- 2. If $\mathbf{V}: \mathcal{P} \rightarrow \operatorname{Hom}_{\mathcal{E}}(1, \Omega)$ is any valuation (see 5.3.9.) (here $\mathcal{P}$ is the set of sentences in the language $\mathcal{L}_{\mathcal{E}}, 1$ is the final object is $\mathcal{E}$, and $\Omega$ is the subobjects classifier from $\left.\mathcal{E}\right)$, then ${ }^{*} \mathbf{V}\left({ }^{*} \mathrm{p}\right)=\mathbf{V}(\mathrm{p})$ for any $p \in \mathcal{P}$ (here ${ }^{*} \mathbf{V}:{ }^{*} \mathcal{P} \rightarrow{ }^{*} \operatorname{Hom}_{*} \mathcal{E}\left(1,{ }^{*} \Omega\right)$ is the non-standard image of the original map $\mathbf{V}, 1={ }^{*} 1$ is the final object, and ${ }^{*} \Omega$ is the subobjects classifier from ${ }^{*} \mathcal{E}$ );

- 3. For any $X \in O b(\mathcal{E})$ and any finitely satisfiable relation $R$ on $\mathrm{X}$ there is $g \in{ }^{*} \operatorname{Hom}_{*} \mathcal{E}\left(1,{ }^{*} X\right)$ such that $\alpha^{*} R g$ for any $\alpha \in H o m_{\mathcal{E}}(1, X)$ (we say that any finitely satisfiable relation is globally satisfiable) (if $R \subseteq \operatorname{Hom}_{\mathcal{E}}(1, X \times X)$ then ${ }^{*} R \subseteq{ }^{*} \operatorname{Hom}_{*} \mathcal{E}\left(1,{ }^{*} X \times{ }^{*} X\right)$ ).

Definition 6.2.4. The inequality ${ }^{*} \mathbf{V}\left({ }^{*} p\right) \leq \mathbf{V}(p)$ from 2 . is called Standardisation (S), the converse inequality is called Transfer (T), and the propriety 3 . is called Idealization (I). They generalize the similar proprieties from CL appearing in 6.1., and can be formalized in the apropriate language.

Theorem 6.2.5. For any SET - type topos $\mathcal{E}$ (see 5.2.4.) there is a non-standard extension (in the sense of 6.2.3.) $*: \mathcal{E} \hookrightarrow{ }^{*} \mathcal{E}$.

Proof. Step 1. Let's consider the topos $\mathrm{SET}^{n}$ having as objects n-uples of sets, and as morphisms n-uples of fumctions, with the canonical compositions and identities, defined component-wise. At this step we will built a non-standard extension for this particular topos. This will be the topos ${ }^{*} \mathbf{S E T}^{n}$, having as objects n-uples $\left({ }^{*} A_{1}, \ldots,{ }^{*} A_{n}\right)$ where $\left(A_{1}, \ldots, A_{n}\right)$ runs through the objects of $\mathbf{S E T}{ }^{n}$, and as morphisms n-uples of internal functions $\left({ }^{*} f_{1}, \ldots,{ }^{*} f_{n}\right)$, where $\left(f_{1}, \ldots, f_{n}\right)$ runs through the morphisms of $\mathrm{SET}^{n}$ (with * being the usual non-standard extension from 6.1). Due to the propriety of transfer (T) of* (see 6.1) and because of 6.1.3., we get that all the proprieties of $\mathbf{S E T}^{n}$ contributing to its structure of topos transfers to ${ }^{*}$ SET ${ }^{n}$. In particular, $\Omega_{* \mathbf{S E T}^{n}}={ }^{*} \Omega_{\mathbf{S E T}^{n}}=(\{0,1\}, \ldots,\{0,1\})$ (so both topoi have $2^{n}$ truth values).

Then $\mathrm{SET}^{n} \stackrel{*}{\hookrightarrow}{ }^{*} \mathrm{SET}^{n}$ and this is a conservative extension of topoi.

More precisely, one can build a NSA on $\mathrm{SET}^{n}$ by imitating the case SET. Namely, in SET ${ }^{n}$ we define a union, intersection, and power set on components, namely :

$$
\begin{aligned}
& \left(A_{1}, \ldots, A_{n}\right) \cup\left(B_{1}, \ldots, B_{n}\right):=\left(A_{1} \cup B_{1}, \ldots, A_{n} \cup B_{n}\right) \\
& \left(A_{1}, \ldots, A_{n}\right) \cap\left(B_{1}, \ldots, B_{n}\right):=\left(A_{1} \cap B_{1}, \ldots, A_{n} \cap B_{n}\right) \\
& \mathcal{P}\left(A_{1}, \ldots, A_{n}\right):=\left(\mathcal{P}\left(A_{1}\right), \ldots, \mathcal{P}\left(A_{n}\right)\right) .
\end{aligned}
$$

Then we can build an Universe $\mathcal{U}(X)$ using the same procedure as in 5.1.12. (here $X=$ $\left.\left(X_{1}, \ldots, X_{n}\right)\right)$. In fact, we get that $\mathcal{U}(X)=\left(\mathcal{U}\left(X_{1}\right), \ldots, \mathcal{U}\left(X_{n}\right)\right)$, where $\mathcal{U}\left(X_{i}\right)$ are Universes as in 5.1.12. We can also build the non-standard extension $\mathcal{U}(X) \stackrel{{ }^{*}}{\hookrightarrow} \mathcal{U}\left({ }^{*} X\right)=\mathcal{U}\left(\left({ }^{*} X_{1}, \ldots,{ }^{*} X_{n}\right)\right)=$ $\left(\mathcal{U}\left({ }^{*} X_{1}\right), \ldots, \mathcal{U}\left({ }^{*} X_{n}\right)\right)$.

We extend the language to $\mathcal{L}_{\mathcal{U}(X)}$ (5.1.13.) and we obtain that $\mathcal{L}_{\mathcal{U}(X)}=\left(\mathcal{L}_{\mathcal{U}\left(X_{1}\right)}, \ldots, \mathcal{L}_{\mathcal{U}\left(X_{n}\right)}\right)$; this can be further component-wise extended to $\mathcal{L}_{\mathcal{U}\left({ }^{*} X\right)}$.

With 6.1.4. in mind, one can define in $\mathrm{SET}^{n}$ the notions of standard and non-standard object on components (the standard objects are of the form $\left({ }^{*} X_{1}, \ldots,{ }^{*} X_{n}\right)$, with $X_{1}, \ldots, X_{n}$ sets; the non-standard objects have an hierarchy of non-standardness, depending on how many components are 
non-standard, and which ones - one can rank them using the lexicographical order on n-uples); one can define, for the non-standard objects, the internal and the external objects, namely an object $\left(X_{1}, \ldots, X_{n}\right)$ is internal iff all the sets $X_{i}$ are internal; and, $\left(X_{1}, \ldots, X_{n}\right)$ is external iff at least one of the $X_{i}$ is external (as in the case of the non-standard sets, one gets several degrees of externality, lexicographically ordered). A sentence in $\mathcal{U}(X)$ consists of an n-uple of sentences from $\mathcal{U}\left(X_{1}\right), \ldots, \mathcal{U}\left(X_{n}\right)$, in this order. The degree of truth of a sentence in $\mathcal{U}(X)$ depends on the number of the true sentences from the components, and on their order, with the lexicographical order on n-uples. The degree of truth of a sentence $\phi$ in $\mathcal{U}(X)$ is kept in $\mathcal{U}\left({ }^{*} X\right)$ and vice-versa, due to $T$ (transfer) and $S$ (standardisation) from the NSA on SET. We got in such a way a conservative extension of the intuitionistic ( $2^{n}$ valued) $\operatorname{logic}$ of the topos $\mathrm{SET}^{n}$. The idealisation property $(I)$ from NSA remains unchanged.

Step 2. It is easy to see that $\mathbf{S E T}^{n}=\mathbf{S E T}^{\mathbf{n}^{o p}}$, with $\mathbf{n}$ from 5.2.3. 2) (note that $\mathbf{n}$ is self-dual).

Step 3. Cf. 5.2.4. a category of SET-type has the form $\mathbf{S E T}{ }^{\mathcal{C}^{o p}}$, with $\mathcal{C}$ finite (that is having a finite number of objects and morphisms), so, there is $n \in \mathbb{N}$ such that $\mathbf{n}$ is a subcategory of $\mathcal{C}^{o p}$ ( $n=$ $\left.\operatorname{card}(O b(\mathcal{C}))=\operatorname{card}\left(O b\left(\mathcal{C}^{o p}\right)\right)\right)$. So, our SET-type category is a subcategory of $\mathbf{S E T}^{n}=\mathbf{S E T}^{\mathbf{n}}$. Now, it is not hard to check that the restriction of the monomorphism * from Step 1 gives a non-standad extension of our SET-type topos.

\section{Infons, Energons, and Receptons : Mathematical Definitions}

This is a concluding section of our study, based on all of the previous work. In 7.1. we define the intuitionistic context, and in section 7.2. we define mathematically the infons, receptons, and energons, providing some examples of receptons.

\subsection{The intuitionistic context.}

Definition 7.1.1. A set is a class which is an element of another class (see 5.1.1.). Let's denote by $\mathcal{M}$ the class of sets. The intuitionistic set theory $(I Z F)$ is the triplet $\left(\mathcal{L}_{\mathcal{M}}, I L+I Z F, \mathcal{D}=\{M P\}\right)$, where $I L$ are the axioms from 5.3.1., $M P$ is as in 5.1.6., and $I Z F$ (Intuitionistic Zermelo-Fraenkel) consists in the following axioms (recall that " $\in$ " and "=" are primary relations)

$I Z F$ : Axioms of ZF (see 5.1.9. and 5.10 - 5.13 from [25]), where the axiom Regularity (R) is replaced by the weaker axiom (Induction):

$\forall x((\forall y \in x \wedge \phi(y)) \rightarrow \phi(x)) \rightarrow \forall x \phi(x)$, for every formula $\phi$.

Note that this in setting the double negation, de Morgan's relations, and tertium non datum, do not hold.

Definition 7.1.2. An Intuitionistic Theory is an usual theory $\mathcal{T}=(\mathcal{L}, \mathcal{A}, \mathcal{D})$ (see 5.1.7.) in an intuitionistic context, so assuming that $\mathcal{A}$ contains the axioms $I L$ and $I Z F$ from before, and that $\mathcal{D}$ $=\{M P\}$ (see 5.1.6.). As in 5.1.7., we assume, by definition, that a theory has at least one model.

Definition 7.1.3. Using the same notations as in 6.1.1., but with IZF from 7.1.1. for intuitionistic set theory, we denote by IIST $=\mathrm{IZF}+\mathrm{IL}+\mathrm{I}+\mathrm{S}+\mathrm{T}$, with IL from 5.3.1., and I,S,T as in 6.2.4. As in 6.1.1. we define the Theory of Intuitionistic Non-standard Extensions, replacing IST by IIST, and taking only $\mathcal{D}=\{M P\}$ from 5.1.6. The propositional calculus of this theory is a conservative extention of the 
intuitionistic logic (that is by $\mathrm{T}$ and $\mathrm{S}$ one conserve the truth values).

\subsection{Infons, Receptons, Energons.}

Definition 7.2.1. We call a generalized infon a Theory $\mathcal{I}=(\mathcal{L}, \mathcal{A}, \mathcal{D})$ in the context of IIST. We call infon the collection of entities of the previous form, whose category of models form a non-standard enlargements of a topos of the form $\mathrm{SET}^{\mathcal{C}^{o p}}$, where $\mathcal{C}$ is any category (see Section 5. also). We call preliminary defined infon the infon whose associated category is a non-standard enlargement of a topos of SET-type (see 5.2.4.).

Definition 7.2.2. We call a generalized recepton the category of models of a generalized infon. We call recepton any non-standard enlargement of a topos of exponential type - SET ${ }^{\mathcal{C}^{o p}}$, where $\mathcal{C}$ is any category (see Section 5. also). We call preliminary defined recepton any non-standard enlargement of a topos of SET-type (see 5.2.4.).

We recall in the context that the logic from topoi is intuitionistic, and its non-standard enlargement is a conservative extension (see Sections 5. and 6.), so the infon and the recepton are compatible entities, representing the two faces of the same entity.

Remark-Definition 7.2.3. An energon is also generalized, normal and preliminary, as before. It is partially infon (when dealing with theories related to potential energy) and partially recepton (when dealing with the kinetic, caloric, electromagnetic actual energies), see also Section 3.

Examples 7.2.4. (of preliminary defined receptons) 1).By definition, any non-standard enlargement of a SET-type topos is a recepton. Particular receptons are represented by the enlargement of the graph-type topos $\mathbf{S E T}_{\overrightarrow{\mathcal{G}}^{o p}}$, or by the enlargement of a geometric topos (see 5.2.6. and 5.2.7.).

2).The Nicolas Bourbaki group of prominent mathematicians from France considered that the fundamental structures in mathematics are (both in the language of theories and of categories) the algebraic structures, topological structures, and order structures, all the others (like the differential ones, for example) being combinations between them. So, in order to apply Mathematics in various arias, one needs to consider collections of such structures, communicating between them. This means that we need to consider various theories and categories together. In the case of categories, the structures are collected in categories, and the communication between them is done with the help of the functors. This means that, in fact, one needs to work with categories of type CAT (see 5.1.21.), and, as a consequence, with appropriate infons and receptons. This means that an important family of receptons will have the form SET $^{\text {CAT }^{o p}}$ and similarly for the corresponding theories. For example, in Multiversal Quantum Physics several mathematical structures appearing in the usual Quantum Physics were put together in such kind of topoi, such as Hilbert spaces with compact self adjoint operators, von Neumann algebras, and others (see [12]). However, when studying the human and global brain, is necessary to enlarge the things to the non-standard environment, due to the role played by the subconsciousness (see [26], [25]).

\section{Conclusions and Further Considerations}

Concluding, in this paper we get three kinds of contributions. One is related to pure mathematics by constructing non-standard analysis in the SET-type topoi (Theorem 6.2.5.). Another one is the presentation of theories from Physics, Neuroscience, Cybernetics focused on the importance and 
appearence of the Infons, Energons, Receptons: how they should look like and which is their usefulness (Sections 2.,3.,4.). And, finally, the interpretation of some mathematical theories (Sections 5.,6.) in terms of infons and receptons, in section 7., compatible with the sections 2.,3.,4.

We will continue our work with part II, where we will build non-standard analysis in general exponential topoi, with applications to infons and receptons in their natural form. Then, these notions should be used in concrete problems of Physics, Neuroscience, Cybernetics, Artificial Intelligene, Economics,... In particular, we will study the (quantum)neural networks and (quantum) Turing machines in the context of topoi, related to the interplay brain/mind (genetic/epigenetic) and in connection with the global brain.

8.1. Example. We end this paper noting that the theory IIST from Section 7. (ZF from Section 5. in the classical case) represents an Infon, containing the empty set. From it (so, from nothingness), one built all the mathematics, by receiving the theory through various Receptons, parts of the Global Brain. And by the Tegmark's Axiom for Multiverses of rank 4 (see 3.1) all the physical entities progressively appear, through Consciousness.

Aknowledgement. The authors of this paper had fruitful scientific discussions with Prof. Leon Zăgrean (Faculty of General Medicine, Bucharest) and Dr. Florin Nichita (Simion Stoilow Institute of Mathematics of the Romanian Academy).

\section{References}

1. R.M. Anderson, Non-standard analysis with applications to economics, in Handbook on Mathematical Economics, vol IV, Elsevier, 1991.

2. C.N. Bodea, I.R. Badea, Optimizing Project Time-Cost-Quality by Using Genetic Algorithms, No 34, Proceedings of the 4th International Conference on Knowledge Management: Projects, Systems and Technologies,Bucharest, November 2009.

3. I.R. Badea, E-learning Tools for IT Project Teams, The 15th International Scientific Conference on Software for Education, Bucharest, April 2019.

4. C.N. Bodea, I.R. Badea, R.I. Mogos, A Multi-Agent System with Application in Project Scheduling, Management and Marketing Challenges for the Knowledge Society, Vol. 6, No. 4(2011) pp. 573590.

5. E. Bomarshenko, The Landauer Principle: Re-Formulation of the Second Thermodynamics Law or a Step to Great Unification ?, Entropy 2019, 21, 918, MDPI, pp. 2-6.

6. M.J. Brockway, Topoi and Logic, M.Sb. Thesis, Victoria University of Wellington, 1976.

7. C.C. Chang, H.J. Keisler, H.J. Model Theory, 3rd ed., Studies in Logic 73(1992), North-Holland.

8. A. Doring, C,Isham, Classical and Quantum Probabilities as Truth Values, arXiv:1102.2213v1 quant-ph, 2011.

9. M. Drăgănescu, The Information of the Matter (in Romanian), Editura Academiei Romne, 1990, Bucharest, Romania, p. 23

10. D. Eagleman, The Brain : The Story of You, Canongate Books Ltd., 2015.

11. H. Everett, The Theory of the Universal Wavefunction, in Many-worlds Interpretation for quantum mechanics, Princeton Univ. Press, 1979, pp. 3-140. 
12. C. Flori, Lectures on Topos on Quantum Theory, Graduate Course 2012, Arxiv 1207.1744, 2012.

13. R. Goldblatt, Topoi: the Categorial Analysis of Logic, North-Holland, 1984.

14. R. Goldblatt, Lectures on the Hyperreals, Springer, 1998.

15. M. Golu, Principles of Cybernetic Psychology, Editura tiinific i enciclopedic., 1975, Bucharest, Romania, 314p.

16. M. Golu, The Neurophysiological Bases of the Psychic (in Romanian), Bucharest, Romania, 1981.

17. B. Greene, The Elegant Universe, W.W.Norton, 1999.

18. Y. Gurevich, I. Neeman, The logic of infons: the Propositional Case, ACM Transactions on Computational Logic, Vol. 12, No. 2 (2011).

19. N. Haramein, The Resonnance Project, https://resonancescience.org/.

20. F. Heylighen, Principles of Systems and Cybernetics: an evolutionary perspective, http://pespmc1.vub.ac.be/Papers/PrinciplesCybSys.pdf.

21. T. Hunt, J.W. Schooler, The Easy Part of the Hard Problem: a Resonance Theory of Consciousness, Frontirs in Human Neuroscience), October 2019, DOI 10.3389/fnhum.2019.00378.

22. T.M. Jessel, E.R. Kandel, J.H. Schwartz, Principles of Neural Science, 3rd ed. Appleton and Lange, 1991, ISBN0-8385-8068-8.

23. N. Jurcău, Two Specialists in Cybernetics: Stefan Odobleja and Norbert Weiner. Common and Different Features, Technical University, Cluj-Napoca, http://www.bu.edu/wcp/Papers/Comp/CompJurc.htm

24. D.K.F. Meijer, I. Jerman, A.V. Melkikh, V.I. Sbitnev, Consciousness in the Universe is Tuned by a Musical Master Code: A Hydrodynamic Superfluid Quantum Space Guides a Conformal Mental Attribute of Reality. The Hard Problem in Consciousness Studies Revisited, Preprint, Research Gate, December 2019, https://www.researchgate.net/publication/338147415.

25. C.-E. Mocanu, F.F. Nichita, O. Păsărescu, O. Applications of Non-Standard Analysis in Topoi to Mathematical Neuroscience and Artificial Intelligence: I. Mathematical Neuroscience, Preprints 2020, 2020010102 (doi: 10.20944/preprints202001.0102.v1).

26. G. Northoff, N. Tsuchiya, H. Saigo, Mathematics and the Brain: A Category Theoretical Approach to Go Beyond the Neural Correlates of Consciousness, Entropy, 21(12), 1234, 2019, https://doi.org/10.3390/e21121234.

27. S. Odobleja. Introduction to the Logic of Resonance (in Romanian), Ed. Scrisul Romanesc, 1984, Bucharest, Romania.

28. S. Odobleja, Diversity and Unit in Cybernetics, 4th Congress of Cybernetics and Systems, at Amsterdam, 21-25 August 1978.

29. O. Păsărescu, On the Classification of Singularities of Normal Gorenstein Surfaces with Ample Anticanonical Divisor, (in Romanian), St. Cerc. Mat., 36(1984), pp. 227-243.

30. O. Păsărescu, O., The Classification of Singularities of Degenerate del Pezzo Surfaces and Structure Theorems, Preprint IMAR, 1981.

31. A. Păsărescu, A., O. Păsărescu O. Study on Non-standard Convergent Series, An. Univ. Pitesti, 1999.

32. A. Păsărescu, Nonstandard Algebraic Methods in the Study of Analytic Spaces, (in Romanian), Ph.D. Thesis, Geometry Balkan Press, 2003. 
33. N. Popescu, L. Popescu, Theory of Categories, Ed. Acad., Sijthoff and Noordhoff, 1979.

34. A. Radu, Topoi Theory, (in Romanian), Romanian Academy Publishing House, vol I 1981, vol II 1982.

35. A. Robinson, Non-standard Analysis, Princeton University Press, 1974.

36. S. Rudeanu, Boolean function and Equations, North Holland, 1974.

37. A. Seth, 30-Seconds Brain, Ivy Press Limited, 2013.

38. J.D. Siegel, Mind : A Journey to the Heart of Being Human, Mind Your Brain Inc., 2017.

39. T. Stonier, Information and the Internal Structure of the Universe, Springer-Verlag, 1990.

40. R. Street, Lectures in Topos Theory, Monash University, 1974.

41. M. Tegmark, Our Mathematical Universe: My Quest for the Ultimate Nature of Reality, Knopf, 2014.

42. N. Tesla, On Electricity, The Address On the Occasion of the Commemoration of the Introduction of Niagara Falls Power In Buffalo At the Ellicot Club, January 12, 1897), Electrical Review, January 27, 1897.

43. The Impact of Nikola Tesla's Patents to Development of Modern Mobile and Internet Services, February 2019, DOI: 10.1109/EnT.2019.00007, Conference: 2019 International Conference on Engineering Technologies and Computer Science (EnT), Bozidar Radenkovic, Sergei Prokhorov, Marijana Despotovic-Zrakic, Zorica Bogdanovi, Aleksandra Labus.

44. J. Walleczek, G. Grossing, P. Pylkkanen, B. Hiley, eds Emergent Quantum Mechanics, 2019, www.mdpi.com/journal/entropy.

45. N. Wiener, Cybernetics: Or Control and Communication in the Animal and the Machine, Paris,(Hermann and Cie) and Camb.Mass. (MIT Press), 1948. 